\title{
Intellectual effects of delayed playback task and rhythmic activities
}

\author{
Kazue Sawami ${ }^{*}$, Himeyo Nakagawa ${ }^{1}$ and Chizuko Suishu ${ }^{2}$ \\ ${ }^{1}$ Nara Medical University, Japan \\ ${ }^{2}$ Shubun University, Japan
}

\begin{abstract}
Introduction: We conducted preventive interventions for aging-associated cognitive decline (AACD), and compared cognitive ability before and after the intervention. Furthermore, an anteroposterior comparison of health-related quality of life (HR-QOL) and mood states was carried out between the intervention and control groups. We also determined the correlation between cognitive ability, HR-QOL, and mood state.

Materials and methods: We recruited 100 elderly people by distributing public relations magazines and leaflets. For the first three months, the participants attended the seminar as a control period. In the subsequent three months, the intervention using the delayed playback task and rhythmic activities was carried out. The tool used to test cognitive ability was the Five Cognitive Functions (Five Cog), which was developed as a screening test of AACD. The tool used for measurement of HR-QOL was the WHO Quality of Life 26, while the Profile of Mood States-Brief form was used for measurement of mood states. Analysis was conducted with t-test corresponding to the comparison between the intervention group and the control group. Spearman's rank correlation coefficient was used for the relationship among each of test items.

Result: The results of the Five Cog revealed that $60.4 \%$ had no cognitive impairments, 37.7\% showed suspected AACD, and $1.9 \%$ showed suspected dementia. In the pre-post comparison of cognitive abilities, the total score significantly improved ( $p<0.05)$. HR-QOL also improved overall and in terms of physical, psychological ( $p$ $<0.01$ ), and social QOL $(\mathrm{p}<0.05)$. For mood states, tension-anxiety, depression-dejection, fatigue-inertia, and confusion-bewilderment all showed reduced scores at post-test, while vigor-activity improved $(\mathrm{p}<0.01)$. There were positive correlations between HR-QOL and cognitive ability in terms of the attention domain of cognitive ability and psychological QOL, and the reasoning domain and social QOL $(\mathrm{p}<0.05)$. There were negative correlations between mood states and cognitive ability - between the reasoning domain of cognitive ability and tension-anxiety, and between the verbal fluency domain confusion-bewilderment ( $\mathrm{p}<0.05$ ).

Conclusion: Cognitive ability was positively related to psychological and social QOL, and negatively related to tension-anxiety, depression-dejection, and confusionbewilderment. Accordingly, a key aspect of interventions for AACD should be promotion of psychological stability and social interaction. This would apply to all such interventions that relate to the interventions for AACD.
\end{abstract}

\section{Introduction}

According to the World Alzheimer Report, the number of dementia patients is increasing exponentially, with the estimated 44 million at present expected to triple by the year 2050 [1]. Part of this emergency situation that has been attracting particular attention is aging-associated cognitive decline (AACD). The criteria for AACD were created by the International Psychogeriatric Association in 1993.

AACD refers to difficulties in any one of the following areas: memory and learning; attention and concentration; thinking (e.g., problem-solving, abstraction); language (e.g., comprehension, word finding); and visuospatial functioning [2]. Subjects classified as AACD have a $28.6 \%$ conversion rate to dementia over 3 years (relative risk $=21.2$ ). In the general population, the prevalence of AACD has been found to be $19.3 \%$ [3].

Against this backdrop of AACD, we carried out a preventive intervention. We then analyzed subjects using anteroposterior comparison of their cognitive ability to measure the effect of the intervention. Furthermore, anteroposterior comparisons between health-related quality of life (HR-QOL) and mood states were carried out in both the intervention and control groups. It was predicted that mental condition and HR-QOL both would influence cognitive ability, so we confirmed the correlations between these variables because this has not been done previously.

\section{Materials and methods}

1. We recruited 100 elderly people by distributing public relations magazines and leaflets. For the first three months, the participants attended the seminar as a control period. In the subsequent three months, the intervention using the delayed playback task and rhythmic activities was carried out.

The delayed playback task was carried out using a brain training game method in small groups as a means of improving declining ability with aging when remembering events of a short time ago. The rhythmic activities were carried out to improve cerebral blood flow and to obtain a mentally-refreshed feeling.

Correspondence to: Kazue Sawami, Ph.D., Principal Investigator, Gerontological Nursing, Nara Medical University, 840 Shijo-cho, Kashihara, Nara 634-8521, Japan; Tel: 81-744-22-3051; E-mail: sawami@naramed-u.ac.jp

Key words: AACD, health-related quality of life, mood states, delayed playback task, rhythmic activities

Received: May 03, 2017; Accepted: May 22, 2017; Published: May 25, 2017 
2. The tool used for testing cognitive ability was the Five Cognitive Functions (Five Cog).

The Five Cog is unique in that it can be administered in a group setting and average scores can be obtained by age and years of education. It was developed as a screening test for AACD. Scores of 15 or above indicate no cognitive impairment after calculating by age, years of education, and gender; 11-14 indicates the possibility of AACD; and less than 10 indicates the possibility of dementia [4].

This tool to measure cognitive domains consists of the hand movement (finger task), position judgment task (attention), word memory task (memory/learning), clock drawing task (visuospatial function), animal name imagination task (verbal fluency), and common word task (reasoning).

3. The tool used to assess HR-QOL was the Japanese version of the WHO Quality of Life 26 (WHO-QOL26). This is composed of 24 items assessing four domains of QOL: physical, psychological, social, and environmental. Furthermore, it contains two additional items evaluating overall QOL, for a total of 26 items. Subjects respond to the 26 items in terms of how they felt in the past two weeks and how satisfied they were in the past two weeks on a 5-point scale: "not at all," "just a little," "somewhat," "very much," and "extremely."

4. The tool used for measurement of mood states was the Japanese version of the Profile of Mood States-Brief form (POMS). This uses six scales- "tension-anxiety," "depression-dejection," "anger-hostility," "fatigue-inertia," "vigor-activity," and "confusion-bewilderment"- to measure individuals' feelings and emotional state. There are a total of 30 items, and subjects respond on whether they experienced each item in the preceding week on a 5-point scale: "not at all," "just a little," "somewhat," "very much," and "extremely."

\section{Period}

October 2015 - April 2016

\section{Statistical analysis}

Paired t-test was used to examine the effects of the intervention on participants in the intervention group by comparing the variables before and after the intervention. Then, the relationship between cognitive ability, HR-QOL, and mood states was analyzed using the Spearman's rank correlation coefficient.

\section{Ethical considerations}

The outline of the research and participants' freedom to refuse, right to anonymity, and agreement as to the publishing of results were explained to prospective participants, both in writing and verbally, and their informed consent was obtained. This study protocol was approved by the ethical review boards of the institution of Nara Medical University.

\section{Result}

Among the 139 registrants, data of 112 people who were able to participate of the whole process were analyzed. The average age of the subjects is $68.8 \pm 8.8$, with 13 being male and 99 being female.

According to the results of the Five $\mathrm{Cog}, 60.4 \%$ had no impairments in cognitive ability, $37.7 \%$ showed suspected AACD, and $1.9 \%$ showed suspected dementia (Figure 1).

In the pre-post comparison of cognitive abilities as shown in Figure 2, the subscales of the Five Cog improved significantly. There was a

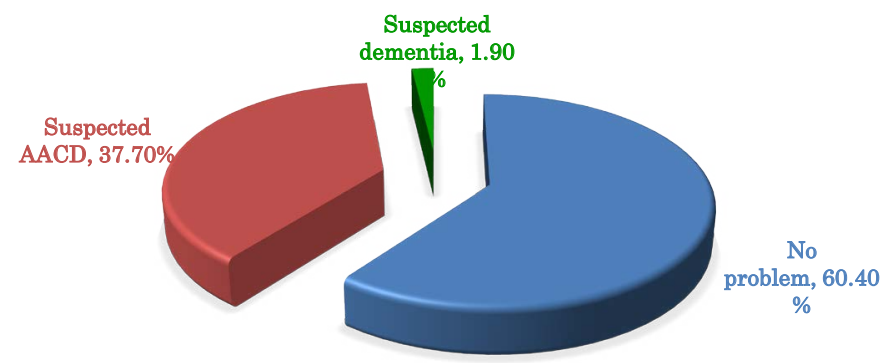

Figure 1. Participants according to cognitive ability (Five Cognitive Functions). ( $\mathrm{N}=112$ )

significant improvement after intervention in hand movement (finger task), position judgment task (attention), word memory task (memory / learning), and common word task (reasoning) ( $\mathrm{p}<0.01)$.

The results of the WHO-QOL26 for the comparison between pre and post-intervention and control are shown in Figure 3; comparing before and after the intervention using the Paired t-test revealed statistically significant increases in overall and the physical, psychological $(\mathrm{p}<0.01)$, and social domains $(\mathrm{p}<0.05)$ of QOL.

With POMS, a significant decrease in tension- anxiety, depressiondejection, fatigue- inertia, and confusion-bewilderment was observed after the intervention, along with a significant increase in vigor-activity $(\mathrm{p}<0.01$, Figure 4$)$.

Significant improvement in cognitive function, mood and HRQOL was seen after intervention compared with the control period results, and correlation coefficients were confirmed as these synergistic effects suggested.

In the correlation between age and cognitive function, the word memory has the strongest correlation, and retention of vocabulary decreases with age.

Next, the correlations between the results of the Five Cog, WHOQOL26, and POMS are shown in Figure 5. Cognitive ability (i.e., the subscales of the Five $\mathrm{Cog}$ ) showed significant positive correlations with the specific domains of the WHO-QOL26: the attention domain of the Five Cog was associated with psychological QOL, while the reasoning domain was associated with social QOL $(\mathrm{p}<0.05)$.

Spearman's rank correlation coefficient, Criterion variables $=$ subscale scores of the Five Cog. ${ }^{*} \mathrm{p}<0.05$

Note that the figure only shows items with significant correlations.

In addition, there were significant negative correlations between the POMS and cognitive ability: namely, between the reasoning domain of the Five Cog and tension-anxiety, and between the verbal fluency domain and confusion- bewilderment $(\mathrm{p}<0.05)$.

\section{Discussion}

The prevalence of AACD in the general population was $19.3 \%$ according to previous studies, but according to the current investigation, the prevalence was $37.7 \%$. Given this suggested increase in the pre-dementia group, it is considered an urgent task to prevent the development of dementia through strategic methods. In this study, we used an intervention that combines delayed playback task and rhythmic activities, which helped to improve participants' total score on a cognitive test. This improvement of cognitive ability was considered an effect of the concurrent improvement of HR-QOL and mood state. 


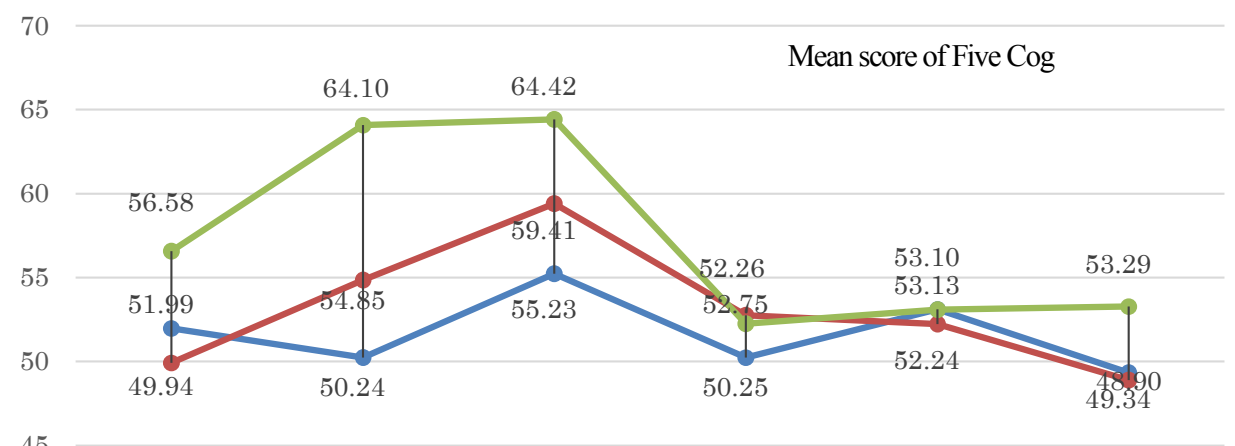

40

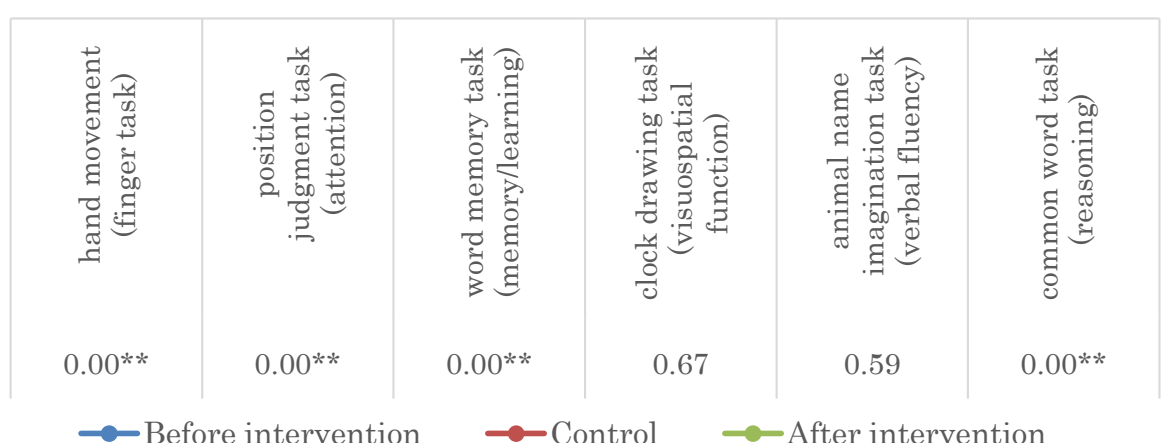

Paired t-test *Significant at the $5 \%$ level, **Significant at the $1 \%$ level.

Figure 2. Pre-post comparison of Five Cogscores $(\mathrm{N}=112)$

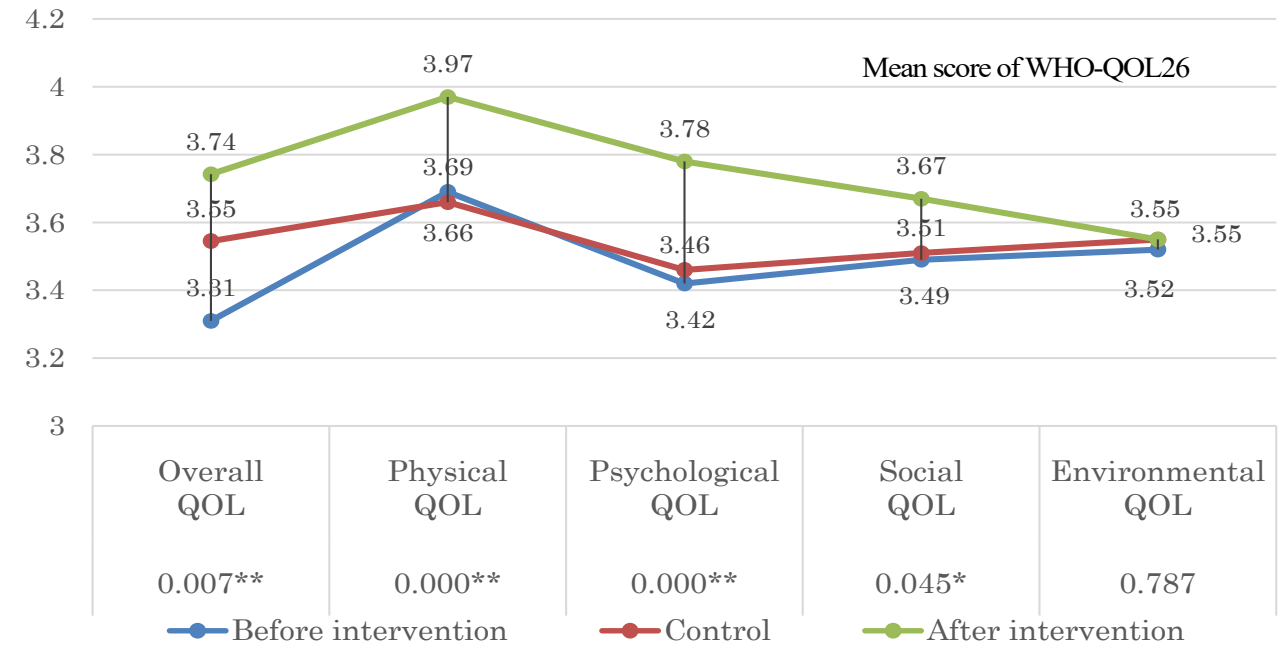

Paired t-test*Significant at the $5 \%$ level, **Significant at the $1 \%$ level.

Figure 3. Pre-post comparison of WHO-QOL26 scores

Cognitive ability was positively correlated with psychological and social QOL and negatively correlated with tension-anxiety and confusion-bewilderment. Accordingly, a key aspect of intervention for managing AACD should be to promote psychological stability and facilitate social interaction. At the start of this intervention, tension-anxiety, depression-dejection, fatigue-inertia, and confusionbewilderment were higher, suggesting that the poor results on the cognitive ability test was due to those factors.
As for non-drug therapies to maintain cognitive functions, most of them are ways such as animal therapy and horticulture therapy to promote pleasant feelings which activate the brain's reward system (A10 nervous system) to become a motivation of keeping up something. In addition, in the study of comparing the effects of positive feelings and negative feelings based on the neutral state, it had been reported that positive feelings expand the ranges of carefulness, thought, and actions while negative feelings reduce the ranges of thought and actions 


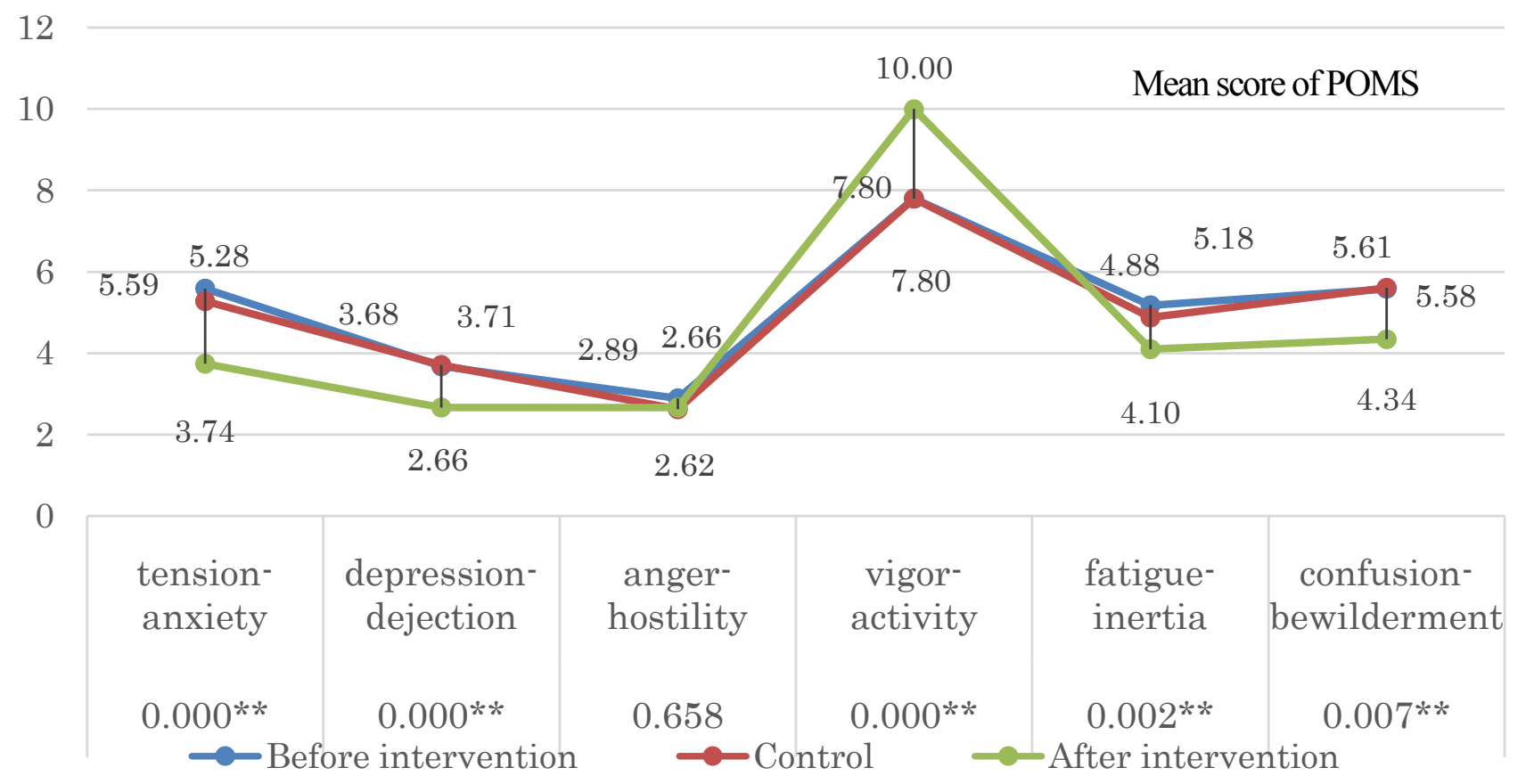

Paired t-test $*$ Significant at the $5 \%$ level, ${ }^{*}$ Significant at the $1 \%$ level.

Figure 4. Pre-post comparison of POMS scores

[5]. We had verified the relationship between eustress/distress and cognitive functions $[6,7]$.

The feature of intervention with the method of playing games in groups is to promote interpersonal exchanges. About improving mood from the promotion of communication among the participants, improving healthy feeling with physical activity, and rise in vigoractivity by involving their familiar activity can be considered as causes of changes to improvement of evaluation results. As of QOL, overall, physical, psychological, social-QOL had significantly increased. By considering the increase in social-QOL, these change occurred not only because of the physical activity and stress prevention but also caused by the communication activity. HR-QOL is known be one factor predicting vital prognosis [8]; in this study, it was suggested to be a main factor affecting cognitive abilities. Moreover, intense psychological stress is known to damage the hippocampus [9], suggesting that the higher the tension, anxiety, depression, or confusion, the greater the reduction in cognitive ability. From this result, it is necessary for an intervention to ensure positive mood states among subjects.

If compare the mental states of participants, particularly psychological-QOL was lower, but after the intervention, overall mental states of participants have increased; especially physical and psychological-QOL has increased larger. About rhythmical exercise adopted in this intervention, that is known to activate serotonin [10, 11]. It is also known that serotonin is related to mental and physical balance and peace of mind as well as influences the memory in the hippocampus and the learning effect.

A characteristic of cognitive decline due to aging is remembering immediately after the event, but not after 20 minutes or so. It is the proposition of this research to collectively prevent this delayed reproduction disorder from the early stage. As a result of this intervention, the delayed reproduction ability decreased with age, but was shown to improve with training.

Until now there has been little to report, although the effects of intellectual activity [12] and social networks have been shown to be defensive factors of the onset of dementia [13]. In the preventive intervention used in this study, the total score for cognitive ability significantly improved after the intervention, and because the related factors of psychological and social QOL and various mood states were demonstrated, we were able to obtain the underlying directionality of the intervention.

\section{Conclusion}

Using an intervention that combines delayed playback task and rhythmic activities, we were able to significantly improve elderly individuals' total scores on a cognitive test. Furthermore, cognitive ability positively correlated with psychological and social QOL, and negatively correlated with tension-anxiety and confusion- bewilderment.

Accordingly, a key aspect of future intervention for managing AACD should be to promote psychological stability and facilitate social interaction.

\section{Acknowledgments}

We would like to thank all the elderly people for participating in the preventative care project. I would like to express my sincere gratitude to the chairman and others of the autonomous association who cooperated in the publicity. In this study, we have demonstrated causal correlations between mood states, cognitive function and other factors that affect quality of life. By forecasting each person's future based on the findings, we seek to provide individualized preventative care programs and to improve subjects' benefits. 

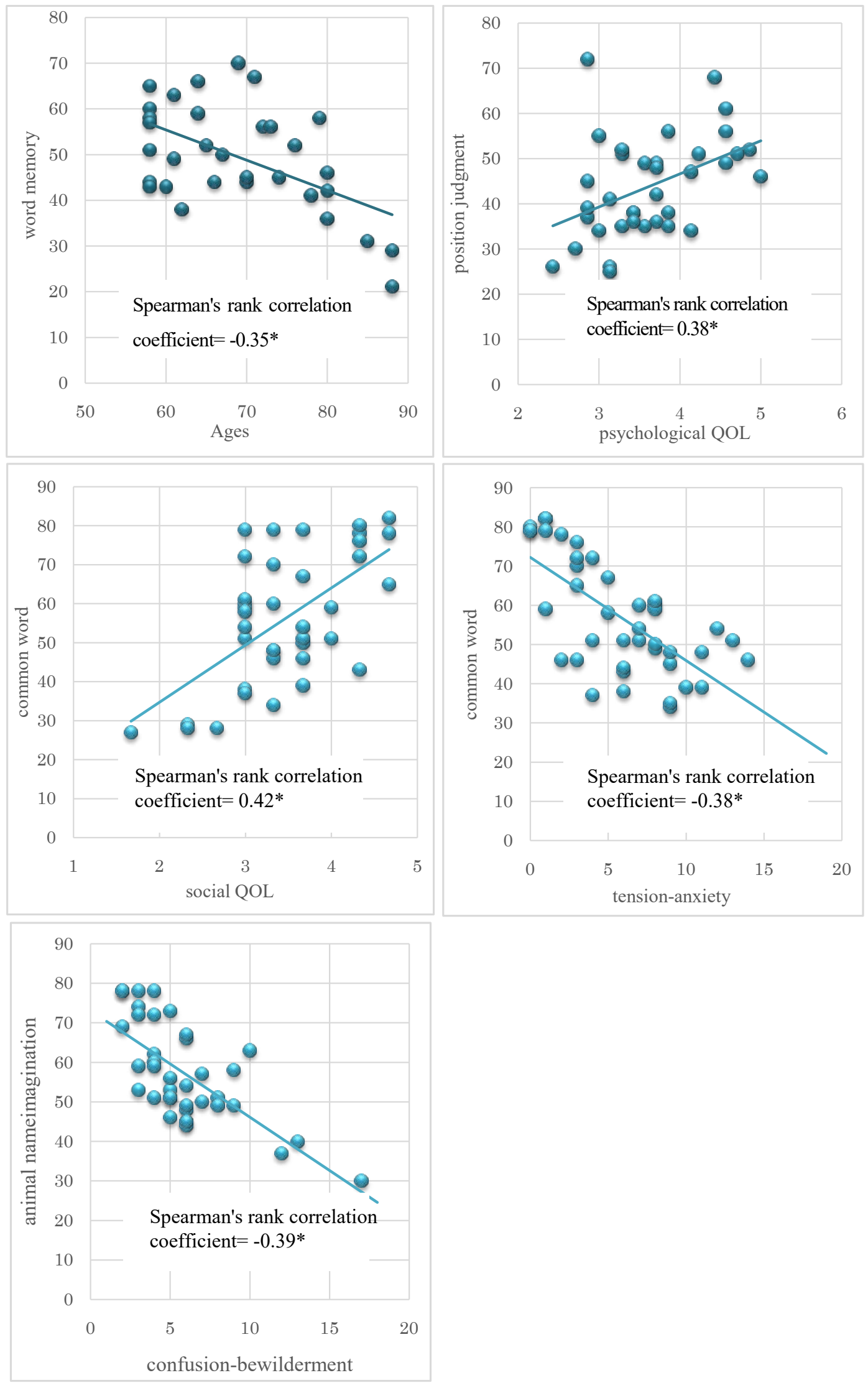

Figure 5. Correlations between the subscales of the Five Cog, WHO-QOL26, and POMS 


\section{References}

1. Alzheimer's Disease International (2013) The global voice on dementia. The Global Impact of Dementia 2013-2050. Alzheimer's Disease International London. 2-7.

2. Levy R (1994) Aging-associated cognitive decline. Working Party of the International Psychogeriatric Association in collaboration with the World Health Organization. Int Psychogeriatr 6: 63-68. [Crossref]

3. Ritchie K, Artero S, Touchon J (2001) Classification criteria for mild cognitive impairment: a population-based validation study. Neurology 56: 37-42. [Crossref]

4. Yatomi N (2010) Collective cognition inspection; Five Cog. Japanese Journal of Geriatric Psychiatry 21:215-220.

5. Fredrickson BL, Branigan C (2005) Positive emotions broaden the scope of attention and thought-action repertoires. Cogn Emot 19: 313-332. [Crossref]

6. Sawami K, Katahata Y, Suishu C, Kamiyoshikawa T, Fujita E, et al. (2017) Examination on Brain Training Method: Effects of n-back task and dual-task. F1000Research 6 .

7. Sawami K, Katahata Y (2017) Report on Wako Association's dementia prevention project: Achievement of activities for prevention of dementia using recreational games. Wako Association's report on implementat 1-12.
8. Otero-Rodríguez A, León-Muñoz LM, Balboa-Castillo T, Banegas JR, RodríguezArtalejo F, et al. (2010) Change in health- related quality of life as a predictor of mortality in the older adults. Qual Life Res 19: 15-23. [Crossref]

9. Akiyama K, Saito A (2006) Stress and Psychiatric Disorders. Dokkyo journal of medical sciences 33: 207-212.

10. Ohmatsu S, Nakano H, Tominaga T, Terakawa Y, Murata T, et al. (2014) Activation of the serotonergic system by pedaling exercise changes anterior cingulate cortex activity and improves negative emotion. Behav Brain Res 270: 112-117. [Crossref]

11. Fumoto M, Oshima T, Kamiya K, Kikuchi H, Seki Y, et al. (2010) Ventral prefronta cortex and serotonergic system activation during pedaling exercise induces negative mood improvement and increased alpha band in EEG. Behav Brain Res 213: 1-9. [Crossref]

12. Wilson RS, Mendes De Leon CF, Barnes LL, Schneider JA, Bienias JL, et al. (2002) Participation in cognitively stimulating activities and risk of incident Alzheimer disease. JAMA 287: 742-748. [Crossref]

13. Fratiglioni L, Wang HX, Ericsson K, Maytan M, Winblad B (2000) Influence of social network on occurrence of dementia: a community-based longitudinal study. Lancet 355 1315-1319. [Crossref]

Copyright: (C2017 Sawami K. This is an open-access article distributed under the terms of the Creative Commons Attribution License, which permits unrestricted use, distribution, and reproduction in any medium, provided the original author and source are credited. 